\title{
Table ronde 4
}

\section{Faut-il de nouveaux \\ modèles économiques \\ ou outils de financement \\ pour accélérer \\ le développement \\ de nouveaux traitements \\ des maladies rares?}

Gilles Roche

Mme Placet, vous consacrez une grande partie de votre temps chez Trophos à chercher des financements. Pourriez-vous nous décrire la vie d'un porteur de projet ou d'un responsable d'entreprise qui doit trouver les moyens de financer ses projets?

\section{Christine Placet}

Trophos est une société de biotechnologie installée à Marseille. Elle en est aujourd'hui au stade des essais cliniques. Nous travaillons sur deux molécules issues de nos propres plates-formes de screening et de développement. Une de ces molécules est aujourd'hui dans un essai pivot de phase II/III sur la SMA (amyotrophie spinale). Nous menons un programme un peu moins avancé sur la sclérose en plaques avec cette même molécule. Avec un autre composé, nous sommes dans une indication de niche en cardiologie.

Notre particularité réside dans notre manière d'avoir réussi à nous financer depuis la création de la société en 1999 jusqu'à aujourd'hui. La question se pose maintenant de savoir comment nous allons continuer à nous financer pour poursuivre nos travaux. Depuis le début de notre existence, nous avons réuni près de 80 millions d'euros. $30 \%$ de cette somme provient de nos actionnaires (qui sont principalement des fonds d'investissement); pour le reste, nous avons fait appel à d'autres sources de financement. En 2000, nous avons conclu un partenariat avec l'AFM, qui finance très largement notre programme sur la SMA. En outre, $30 \%$ de nos dépenses éligibles nous reviennent sous forme de crédit d'impôt recherche I'année d'après. De plus, nous avons réussi à monter des projets collaboratifs qui nous permettent d'aller chercher de l'argent non dilutif auprès des instances nationales, telles qu'Oséo et l'ANR (Agence nationale de la recherche); nous sommes coordonnateurs de deux gros projets européens dans le cadre du $7^{\mathrm{e}}$ programme cadre de recherche et développement (PCRD).

Aujourd'hui, nous devons continuer à trouver des fonds. Le paysage des fonds d'investissement s'est restreint au cours de la décennie passée. Par ailleurs, nous
Participent à la table ronde :

Laurent Arthaud, BPI/CdC Entreprises et Kurma

Virginie Fontaine, Bpifrance

Philippe Gorry, Université de Bordeaux

Patrice Layrac, Angels Santé

Christine Placet, Trophos

Frédéric Revah, Généthon

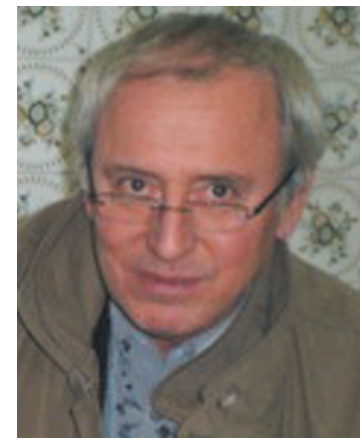

Table ronde animée par Gilles Roche (Eurobiomed)

comprenons que la dimension risquée de notre modèle économique puisse freiner les investissements. Néanmoins, notre capacité à diversifier les sources permet de réduire les risques de chaque investisseur.

\section{Gilles Roche}

Pendant que vous cherchez des fonds, vous n'effectuez pas votre travail de directrice générale.

\section{Christine Placet}

Tout à fait. Trophos est une petite structure, mais nous passons beaucoup de temps et d'énergie à rechercher des fonds.

\section{Gilles Roche}

En pratique, il n'est pas simple de trouver des moyens financiers pour faire de la R\&D pour des start-up ou des PME débutantes dans le domaine des maladies rares. Puisque les ressources sont limitées, il est peut-être judicieux de n'investir que dans certains sujets, comme 
les pathologies particulièrement graves, les produits pour lesquels les chances de succès sont réelles et les produits qui présentent une vraie rupture technologique ou un intérêt économique prévisible plus important. Les investisseurs font le choix d'investir dans tel ou tel secteur économique. Si le modèle économique tient la route et que le futur produit présente des chances significatives d'être rentable, les investisseurs seront sans doute plus enclins à investir. Faut-il présenter à l'investissement certains types de projets dans les maladies rares plutôt que d'autres, de manière à mieux concentrer les ressources?

\section{Philippe Gorry}

Une bonne invention devient une innovation lorsque la société se l'approprie et si elle repose sur un modèle économique qui permet aux investisseurs d'avoir un retour sur investissement. Dans le cadre de la santé, les produits font l'objet d'autorisations, qui sont de plus en plus difficiles à obtenir, à la fois sur les aspects efficacité/sécurité, et sur les aspects médico-économiques. Dans le cadre des maladies rares la preuve de l'innovation n'est pas à faire : les patients sont en attente de traitements. En théorie, le développement dans lequel s'engagent les entreprises est donc plus facile. Celles-ci doivent néanmoins élaborer un modèle économique qui assurera un retour sur investissement pour des populations de malades restreintes. Ce défi économique n'est pas impossible puisque de nombreuses sociétés - en dehors des grands groupes pharmaceutiques - présentent un retour sur investissement intéressant pour les investisseurs. La non-rentabilité économique des produits développés dans le domaine des maladies rares est une image qui ne correspond pas à la réalité. II n'en demeure pas moins que le choix des projets est crucial. Celui-ci doit s'appuyer sur des critères à la fois scientifiques et médico-économiques.

\section{Frédéric Revah}

Le Généthon est un centre de R\&D créé par l'AFM-Téléthon en 1990, avec comme mission de découvrir des médicaments pour le traitement des maladies rares. En 1990, il s'agissait d'abord de participer aux efforts de décryptage du génome humain. Les premières cartes du génome humain ont été découvertes en 1992 par Généthon. Depuis, nous avons évolué de la génomique vers la thérapeutique. Aujourd'hui nous sommes focalisés sur la conception, le développement, la production et les essais cliniques pour des médicaments de thérapie génique. Cette technologie de rupture est particulièrement bien adaptée aux maladies monogéniques et dans $80 \%$ des cas les maladies rares sont d'origine génétique. Comme la conception, le développement et la production de médicaments de thérapie génique diffèrent sensiblement de la conception, du développement et de la production d'une petite molécule; cette technologie de rupture constitue un vrai pari sur l'innovation pour des maladies graves.

Les sociétés pharmaceutiques et les investisseurs classiques ne monttrant pas d'intérêt pour cette approche, l'AFM a décidé de s'y investir pour répondre à un besoin médical qui est réel, même s'il ne répond pas aux exigences de retour financier des intervenants « classiques » du monde pharmaceutique. À ce jour, trois projets sont au stade clinique ; l'an prochain, nous en aurons cinq. Notre stratégie vise donc à nous déployer vers la clinique, avec comme objectif d'amener ces médicaments jusqu'au marché selon un mode non lucratif - puisque nous sommes une association à but non lucratif.

Notre équation économique est très spécifique, car $80 \%$ de notre budget vient des dons du Téléthon. Sans la patience et la persévérance de l'AFM et des donateurs, nous n'aurions pas pu construire ce que nous avons réalisé au cours de nos 23 années d'existence.

Les projets que nous retenons dans le domaine de la thérapie génique sont choisis sur la base du besoin médical et de leur probabilité de réussite. La valeur économique des produits de thérapie génique - et de la recherche sur les maladies rares en général - va largement au-delà de la valeur du produit lui-même. Pour faire une analogie, je dirais qu'aller sur la Lune n'a aucun intérêt économique. En revanche, les retombées de cette aventure humaine sont considérables dans le transport aérien, le domaine spatial, etc. II en va de même pour les maladies rares. Celles-ci constituent en effet un tremplin pour les maladies fréquentes, les premières nous obligeant à mener des innovations de pointe, qui auront des retombées sur les secondes. Nous ferions une erreur majeure si l'analyse économique des maladies rares était restreinte au marché de chaque produit. De toute évidence, la valeur économique globale de la recherche sur les maladies rares doit être appréhendée au-delà du marché spécifique que constitue un produit donné.

\section{Gilles Roche}

Les deux éclairages que nous venons d'entendre sont totalement complémentaires. Ils montrent bien que la vision de santé publique et l'économie privée peuvent intervenir dans une logique de complémentarité. Dans le développement des maladies rares, le coût de R\&D peut-il être réduit en faisant plus d'essais cliniques académiques? Des plates-formes publiques de production pourraient-elles être développées ou mutualisées pour réduire les coûts de production? La partie réglementaire pourrait-elle être allégée pour que les dossiers soient moins coûteux?

\section{Virginie Fontaine}

Bpifrance accompagne des entreprises qui développent des produits d'innovation. Dans le secteur de la santé en 2012 , nous avons financé 430 projets pour 112 millions d'euros dont le développement d'une centaine de médicaments à différents stades (depuis les preuves de concept précliniques non réglementaires jusqu'aux phases cliniques). À travers ce grand volume de projets, nous observons que le marché des maladies rares est 
très attractif pour les PME. La concurrence y est moindre. Les thérapies développées peuvent, par la suite, être adaptées pour des maladies de plus grande ampleur. Le marché est ouvert, notamment parce que les entreprises pharmaceutiques s'en sont désintéressées pendant longtemps.

À travers les projets que nous aidons, les entreprises évoluant dans le secteur des maladies rares optent pour des thérapies extrêmement innovantes - de type thérapie cellulaire ou génique - ou se tournent vers des stratégies de repositionnement de molécules avec AMM qui sont tombées dans le domaine public. La seconde stratégie permet d'accélérer l'accès au marché pour un coût relativement réduit.

\section{Frédéric Revah}

Les expertises de développement dans le domaine des maladies rares et des bibliothèques innovantes (thérapie cellulaire, thérapie génique) sont elles-mêmes rares. La conception d'un essai clinique dans ce domaine est bien différente de celle dans le domaine des maladies fréquentes. Les expertises réglementaires sont également très spécifiques. Pour être efficace, il est donc important de s'appuyer sur des expertises existantes. En France, nous avons la chance d'avoir des acteurs de pointe dans tous les domaines, d'amont en aval, c'est-àdire de la recherche académique au recrutement de patients et à la bioproduction. Pour un des essais cliniques que nous menons sur une immunodéficience rare, il faut savoir que nous avons réussi à recruter cinq fois plus rapidement en France qu'aux États-Unis, notamment grâce aux registres et à un système de soins performant.

En outre, un certain nombre d'acteurs majeurs sont présents en France. Le Généthon est l'un d'eux dans le domaine de la thérapie génique. II est important de structurer ces acteurs en filière, de concentrer les efforts pour dégager des masses critiques et éviter le saupoudrage. La notion de filière est particulièrement importante pour développer de manière plus efficace.

\section{Gilles Roche}

Un scientifique qui démarre une start-up en biotechnologie rencontre d'abord les structures publiques, puis se tourne généralement vers les business angels. Qui sont-ils? Que font-ils dans le domaine de la santé?

\section{Patrice Layrac}

Un business angel n'est pas une personne morale, mais un regroupement de personnes physiques dans une association, voire une société d'investissement. II investit une partie de son patrimoine dans des start-up à potentiel. En outre, il soutient le porteur de projet. Nos membres étant d'origines très diverses (industrie pharmaceutique, hôpitaux, spécialistes de la propriété intellectuelle, financiers, avocats, etc.), nous avons la possibilité de supporter les porteurs de projets dans différents aspects.

Nous investissons dans la phase d'amorçage de sociétés dont nous estimons la valeur comprise entre 500000 et 2000000 euros. Lorsqu'un projet organise plusieurs tours de levée de fonds, nous investissons relativement tôt pour pouvoir éventuellement investir au deuxième tour.
Une autre caractéristique des business angels est qu'ils ont vocation à sortir de l'entreprise dans laquelle ils ont investi. Cette sortie intervient généralement cinq ans après l'investissement initial.

La France compte 80 réseaux de business angels. Régionaux ou thématiques, ces réseaux rassemblent 4400 personnes, qui investissent chaque année 40 millions d'euros dans 350 entreprises (soit un ticket moyen de 115000 euros par entreprise).

Je représente le réseau Angels Santé, qui est le seul réseau français dédié à la santé (pharmacie, service, industrie vétérinaire, etc.). II a créé une société d'investissement de Business Angels (SIBA) qui a permis de lever plus de 1,5 million d'euros de fonds depuis 2011. Nous avons investi dans trois sociétés. Nous cherchons des projets intéressants dans le domaine des maladies rares, et également des projets de repositionnement visant à améliorer des formes galéniques. Nous disposons de 1,5 million d'euros de trésorerie pour investir dans ces projets.

\section{Gilles Roche}

En Languedoc-Roussillon, il existe deux réseaux de business angels. Sud Angels est un peu en veilleuse, tandis que MELIES Business Angels est plus actif. La région PACA compte, quant à elle, sept réseaux de business angels.

Il existe par ailleurs des fonds d'investissement de capital-risque. Deux fonds d'environ 50 millions d'euros, récemment créés, sont dédiés aux maladies rares. Le premier, Kurma Biofund (de KLS Partners), va maintenant vous être présenté par Laurent Arthaud, qui est membre du conseil de surveillance de ce fonds.

\section{Laurent Arthaud}

Le fonds Kurma Biofund est le deuxième fonds créé par l'équipe de Kurma qui travaille depuis une dizaine d'années dans le domaine du capital-risque. Cette équipe est dirigée par Thierry Laugel, Rémi Droller et Vanessa Malier. Ces trois investisseurs chevronnés ont décidé de centrer ce deuxième fonds sur plusieurs domaines, et notamment les maladies rares - du stade d'amorçage au $3^{e}$ tour de financement. Ce fonds de 45 millions d'euros investira dans une douzaine de sociétés. Ses liens avec l'Institut Pasteur et l'Institut Curie sont assez forts.

Le second fonds, qui a également été créé cet été, est le fonds Biothérapies Innovantes et Maladies Rares, que je dirige avec Chahra Louafi. Avec l'AFM-Téléthon, nous avons pris cette initiative, car divers projets financés par l'association doivent à un moment donné sortir de la R\&D et devenir de petites sociétés pour 
développer des thérapies innovantes dans le domaine des maladies rares. Ce fonds d'amorçage concerne les sociétés qui démarrent leurs premières phases cliniques. Doté de 50 millions d'euros, il est financé en partie par l'AFM-Téléthon et les programmes d'investissements d'avenir. Nous envisageons d'investir dans une dizaine d'entreprises.

\section{Frédéric Revah}

L'AFM a contribué, avec la Caisse des dépôts et consignations, à la création du fonds Biothérapies Innovantes et Maladies Rares. Cette initiative est importante, car elle va permettre d'accélérer un certain nombre de projets qui pourraient déboucher sur la création d'une start-up ou d'une entreprise. En investissant dans ces structures naissantes, nous veillons à ce que cette création d'entreprise permette d'accélérer le projet au bénéfice du patient.

La recherche ayant été financée grâce à la générosité du public, le prix final du médicament des sociétés que nous finançons doit répondre à la notion de prix juste et maîtrisé, basé sur la réalité des coûts de $R \& D$, et non pas sur une valeur médicale totalement empirique - qui peut amener aux dérives que nous avons pu observer sur certains médicaments de maladies rares.

\section{Gilles Roche}

Oséo a changé de nom pour devenir BPI France. Mme Fontaine, pourriez-vous nous expliquer l'implication de cette évolution?

\section{Virginie Fontaine}

Bpifrance est une holding regroupant deux filiales: Bpifrance investissement, ex-CDC Entreprise et FSI, et Bpifrance financement, ex-0séo. La première gère en particulier les prises de participations dans les PME. La seconde, qui participe au financement des $P M E$, exerce trois métiers : la garantie, le financement bancaire et l'innovation.

Bpifrance financement aide les entreprises qui innovent à travers des subventions ou des avances remboursables (en cas de succès du programme), encadrées par des régimes notifiés européens. Cette aide permet d'accompagner et de partager le risque pris par les entreprises lorsqu'elles souhaitent développer un programme de R\&D.

Les entreprises qui mènent leur projet seules, afin par exemple de développer un candidat médicament ou réaliser une étude préclinique réglementaire, peuvent recevoir jusqu'à 3 millions d'euros d'aide de notre part.

D'autres entreprises souhaitent développer leur projet dans le cadre d'un consortium réunissant des partenaires publics et privés. Dans le cadre des projets d'innovation stratégique industrielle (ISI), jusqu'à 10 millions d'euros d'aide peuvent être accordés. Dans le cadre des investissements d'avenir, Bpifrance gère les projets de R\&D structurants des pôles de compétitivité (PSPC), qui peuvent concerner aussi bien les PME et les académiques que les grands groupes. Ces programmes de grande ampleur peuvent bénéficier de 50 millions d'euros d'aide.

\section{Gilles Roche}

Les sept pôles de compétitivité santé en France sont là pour contribuer et vous aider à monter des dossiers.

\section{Laurent Arthaud}

La volonté politique était, à travers la partie investissement de Bpifrance, de regrouper les structures d'aide aux sociétés et les structures d'investissement. Sur la partie investissement dans les biotechnologies, il y a le fonds Biothérapies Innovantes et Maladies Rares. Sur la partie fonds de fonds, Bpifrance est souscripteur principal d'une grande partie des fonds existants en France (Sofinnova, BioDiscovery de Rothschild, Auriga...). Bpifrance souscrit pour environ 150 millions d'euros par an dans des fonds de capital-risque, dont plus de la moitié dans les biotechnologies. Nous ne faisons pas uniquement des investissements en direct au sein de Bpifrance - pour ne pas capter tout le marché ; nous cherchons surtout à favoriser l'existence d'une multiplicité de fonds.

\section{Gilles Roche}

Jusqu'à présent, les entreprises pharmaceutiques privées avaient des blockbusters qui rapportaient beaucoup d'argent. Elles réinvestissaient généralement $16 \%$ de leur chiffre d'affaires dans la R\&D. Pourquoi les entreprises privées en général n'autofinancent plus leur R\&D?

\section{Christine Placet}

Les grandes entreprises pharmaceutiques ont diversifié leur façon de faire de la recherche. Une nouvelle manière consiste à acheter des briques en se rapprochant de petites entreprises qui sont à un stade d'avancement dans un développement de médicament suffisant pour les intéresser. Des sociétés comme la nôtre discutent, cherchent et évaluent la possibilité d'avoir un partenariat stratégique avec une big pharma. Un partenariat de ce type nous permettra de financer la poursuite du développement. À l'issue de ce partenariat, il est très probable que notre société soit absorbée par cette grande entreprise pharmaceutique. Dans ce modèle économique intégral, les big pharma sont à la recherche de briques de R\&D bien avancée, et les petites entreprises de biotechnologies arrivent à aller jusqu'au marché, ou se font racheter par une grande entreprise pharmaceutique.

\section{Gilles Roche}

Pour qu'une big pharma soit intéressée par une jeune société, celle-ci doit s'appuyer sur un modèle économique solide. En quoi les maladies rares constituentelles un sujet attractif pour les grandes entreprises pharmaceutiques? 


\section{Philippe Gorry}

La santé correspond à un marché socialement construit, c'est-àdire qu'il ne s'inscrit pas dans une simple logique offre-demande, mais fait l'objet de négociation entre les parties: industrie, état, médecins, patients. II se caractérise par des prescripteurs de valeur (les médecins et, de plus en plus, les agences de santé) qui justifient l'usage d'un traitement pour une condition pathologique. Entre 1950 et 1990, l'industrie pharmaceutique s'est développée sur un marché où il n'y avait pas de réponse thérapeutique à l'ensemble des pathologies. Elle s'est ensuite tournée vers des marchés de substitution, en commençant par se réfugier dans le développement facile de médicaments «me-too». Leurs retours sur investissement étaient très appréciés des investisseurs - l'industrie pharmaceutique était jusqu'il y a peu, plus rentable que le secteur du luxe. À partir des années 1990, l'industrie pharmaceutique a été confrontée à un déficit de produits innovants, qui s'est maximisé au tournant des années 2000 avec des échecs de mise sur le marché de médicaments. Ces entreprises ont alors évolué du modèle de blockbuster à celui de niche-buster. Cette bascule explique pourquoi la quasi-totalité des big pharmas ont aujourd'hui des médicaments orphelins dans leur pipeline. Elles ont donc très bien compris les enjeux. Le retour sur investissement de ces médicaments leur est très favorable, au moins en image de marque.

\section{Gilles Roche}

Les big pharmas ont tout de même mis du temps à comprendre ce changement de contexte.

\section{Philippe Gorry}

Les grandes organisations prennent effectivement toujours plus de temps pour évoluer.

Dans un modèle extrêmement concurrentiel, où le développement médicamenteux est risqué, la solution d'un développement intégralement réalisé en interne est révolue. Les entreprises pharmaceutiques doivent donc intégrer le modèle d'open innovation en apprenant à se ressourcer à l'extérieur en méthodes ou en produits en développement, tout en étant capables d'externaliser tout ou partie de leur recherche et développement (R\&D).

Dans ce contexte, l'ouverture de chimiothèques et le repositionnement de molécules constituent le grand enjeu de l'industrie pharmaceutique de demain, car chacun s'accorde à dire que cette industrie dispose de ressources qui n'ont pas encore été exploitées. Nombre de PME ont d'ores et déjà bien compris cette réalité, car les incitations financières sont au rendez-vous. En outre, la prise en compte de la libération des données cliniques serait source d'opportunités pour la recherche et source de vraies économies.

L'initiative de l'AFM est tout à fait pertinente, car elle s'est engagée dans ce secteur sur le principe de l'économie sociale et solidaire. Ce sujet renvoie à la notion de partage de la valeur. Aujourd'hui, force est de constater que plus l'industrie pharmaceutique se désengage de la R\&D, plus le développement repose sur la force publique. D'aucuns estiment que la nationalisation de
I'industrie pharmaceutique serait très bénéfique aux comptes sociaux de la santé et résoudrait peut-être les conflits des politiques industrielles et de santé publique.

\section{De la salle}

Faut-il allonger la durée des brevets pour les médicaments orphelins?

\section{Philippe Gorry}

Non, je ne crois pas en l'état, car il faut savoir que ces médicaments bénéficient déjà d'une incitation majeure, qui est le statut de médicament orphelin, apparu aux États-Unis dans le Orphan Drug Act de 1983, puis implanté en 2000 en Europe. Ce statut a été inventé pour favoriser le repositionnement de molécules dont le brevet était tombé dans le domaine public et qui pouvaient subvenir au traitement de maladies rares. L'incitation prend la forme d'une exclusivité de marché de sept ans aux États-Unis et de dix ans en Europe, à partir de la date de mise sur le marché du médicament. Depuis 30 ans, moins de $30 \%$ des médicaments ont réellement bénéficié de cette exclusivité de marché. La plus-value de cette disposition est généralement de quatre ans. En outre, il faut savoir que nombre de médicaments orphelins sont des médicaments pédiatriques, qui bénéficient par ailleurs d'autres incitations.

\section{De la salle}

À quel stade de développement d'une molécule une société de biotechnologie est-elle intéressante pour les investisseurs?

\section{Patrice Layrac}

II n'y a pas de réponse à cette question parce que chaque stade de développement peut présenter un intérêt à investir. À chaque étape de développement d'un produit l'entreprise prend de la valeur.

\section{Christine Placet}

La difficulté réside dans le fait que la durée de développement d'un médicament est beaucoup plus longue que la durée d'un investissement dans un projet. Les startup qui se créent ne peuvent pas offrir de sortie à leurs investisseurs avant de nombreuses années.

\section{Gilles Roche}

La valorisation d'une entreprise qui ne réalise pas encore de chiffre d'affaires est loin d'être une science exacte. 


\section{Laurent Arthaud}

Une entreprise est généralement valorisée par la méthode du Discounted Cash Flow (DCF), qui consiste à estimer les revenus futurs de l'entreprise pour ensuite calculer sa valeur actualisée. L'horizon d'une société de biotechnologie est généralement très lointain en matière de trésorerie. Des méthodes de comparable sont généralement utilisées, en intégrant la probabilité pour l'entreprise de mettre son produit sur le marché.

\section{Gilles Roche}

II n'y a pas qu'une seule preuve de concept. Celle-ci peut être uniquement préclinique. On peut aussi considérer que la preuve de concept dans la phase IIA/IIB va vraiment donner de l'intérêt au produit. II est donc important de savoir à quelle preuve de concept il est fait référence. Qui vous amène les projets et à quels stades?

\section{Patrice Layrac}

Nous recevons des projets de la part des entrepreneurs qui nous connaissent, ou via les membres de l'association. Nous participons aussi à des réunions, comme RARE, qui nous permettent de rencontrer des industriels et des entrepreneurs. Sur notre base de données Gust, accessible à tous nos membres, figurent l'ensemble des projets collectés.

\section{Gilles Roche}

Dans le cadre du réseau MELIES Business Angels, nous co-investissons beaucoup avec d'autres réseaux de business angels de PACA, RhôneAlpes, Midi-Pyrénées ou autres. Tous les projets innovants que nous soutenons passent par des incubateurs et des pépinières d'entreprises avec lesquels nous sommes très impliqués.

\section{De la salle}

Quel est le taux de succès des projets que vous recevez?

\section{Laurent Arthaud}

Nous avons réalisé quelques chiffrages sur le capital-risque. La France compte environ 400 sociétés de biotechnologies. En quatre ans, 80 sociétés ont été financées par le capital-risque.

\section{Patrice Layrac}

En 2012, nous avons investi dans dix projets, sur 140 reçus. Après cinq ans, quatre à cinq entreprises ont fait faillite ; deux à trois se sont maintenues (sans profitabilité réelle) et deux entreprises présentent des taux de rendement supérieurs à $40 \%$. Le taux de succès des projets est donc de $20 \%$.

\section{Gilles Roche}

Sur le réseau languedocien, $20 \%$ des dossiers reçus chaque année font l'objet d'une due diligence, et nous investissons dans $4 \%$ des projets reçus. On admet que quelques années plus tard, six entreprises sur dix n'existent plus, trois survivent et une a décollé.

\section{De la salle}

Est-il vraiment possible d'avoir un retour sur un investissement d'une société de biotechnologie qui fait de la R\&D sur des maladies rares après cinq ans?

\section{Christine Placet}

Ce délai est trop court si l'investissement est réalisé à un stade précoce, sauf si une big pharma vient racheter le projet. Si un essai clinique en phase II/III en SMA se traduit par des résultats positifs, nous serons en mesure d'enregistrer dans un horizon de 18 à 24 mois. Pour le financeur qui nous aidera à financer cette phase d'enregistrement, le retour sur investissement sera rapide.

\section{Philippe Gorry}

Aux États-Unis, plus de 1000 jours s'écoulent entre la demande de désignation et l'AMM pour les médicaments orphelins. Un retour sur un investissement après seulement cinq ans est donc très difficile. Rappelons que le délai moyen de mise sur le marché d'un médicament est de 7 à 12 ans. Aussi aux États-Unis, nombre de sociétés de biotechnologie ne développent pas le médicament jusqu'à la mise sur le marché. Elles effectuent les phases intermédiaires de développement, et contractent avec l'industrie pharmaceutique, s'assurant un retour sur investissement potentiellement plus rapide.

\section{Laurent Arthaud}

Les investisseurs ne restent jamais jusqu'à la mise sur le marché des produits par les sociétés de biotechnologie dans lesquelles ils ont investi. II y a toujours une entreprise qui reprend le produit en licence ou qui rachète la société. Le modèle n'est heureusement pas lié au délai de mise sur le marché du produit.

\section{Gilles Roche}

Les maladies rares peuvent être considérées comme un problème de santé publique. Les pouvoirs publics devraient donc jouer un rôle plus important. Quel est le rôle relatif des acteurs privés et publics dans le développement de nouvelles solutions pour les maladies rares?

\section{Frédéric Revah}

Le succès d'un développement dépend de la structuration du projet à travers l'articulation entre des acteurs publics - qui pourront être à l'origine des preuves de concept initiales -, des acteurs privés non lucratifs (comme Généthon, qui assure la «traversée du désert ») et des acteurs privés lucratifs. Ces derniers 
reprendront le projet en main s'il repose sur un modèle économique solide. Pour certaines maladies ultra-rares, il se peut qu'aucun interlocuteur privé ne soit intéressé. L'articulation entre le public et le privé non lucratif est fondamentale.

\section{Gilles Roche}

II n'existe donc pas de stratégie globale permettant de répartir les maladies - et le développement de solutions - entre les acteurs publics, les acteurs privés non lucratifs et les acteurs privés lucratifs.

\section{Frédéric Revah}

Ce modèle reste effectivement à inventer. Une filière doit être cristallisée autour d'un certain nombre d'acteurs forts qui existent en France.

\section{Gilles Roche}

En faisant table rase de l'existant, pourriez-vous décrire quel pourrait être le bon modèle de prise en charge?

\section{Laurent Arthaud}

Entre le début de la chaîne (la R\&D, financée en grande partie par la puissance publique) et la fin de la chaîne (lorsque la puissance publique détermine le prix du médicament), le système ne présente pas forcément une cohérence globale. Or, celle-ci serait très utile pour éviter les «pertes en ligne » actuelles.

\section{Philippe Gorry}

Aujourd'hui, les décideurs politiques sont prisonniers d'un schéma dans lequel ils doivent gérer trois politiques publiques totalement conflictuelles (favoriser la mise sur le marché rapide d'un produit répondant aux besoins des patients ; satisfaire une politique de santé publique et réduire les coûts sociaux; défendre l'emploi de l'industrie pharmaceutique dans le pays en soutenant une politique du prix du médicament). Dans ce contexte, l'innovation sociétale est indispensable pour revoir notre modèle d'ensemble du partage de la valeur. Le développement médicamenteux non lucratif peut être une alternative. Ainsi aux États-Unis, une fondation pour la maladie de Parkinson a créé un fonds de capital-risque pour investir dans la création de sociétés implantées dans le monde entier.

\section{De la salle}

Pour diminuer les coûts, il faudrait mutualiser les infrastructures précompétitives comme cela se fait en Europe avec le programme européen IMI (Innovative Medicines Initiative).

\section{De la salle}

L'open innovation est un concept très important, car nous lancerons notre prochain appel à projets dans ce cadre. Au cours de ce congrès, nous avons vu qu'il existe au moins quatre cohortes d'intérêt pour la communauté française des maladies rares (les cohortes scientifiques pour mieux comprendre l'histoire de la maladie; les cohortes sur des médicaments post-AMM ou hors AMM ; les cohortes médico-économiques; et les cohortes sociétales, sur la qualité de vie des patients). Nous voudrions nous assurer que ces cohortes soient mises en place dès le départ avec un dialogue très fort avec les industriels. Le modèle d'open innovation nous est donc nécessaire. S'il manque des cases dans les données collectées sur ces cohortes de patients, la qualité de l'exploitation des données et du partenariat avec l'industrie en sera affectée. Je suis ravi que le Leem et Ariis aient été inclus dans notre partenariat pour développer le dialogue. L'implication très en amont dans le design des cohortes constitue un sujet fondamental. Le modèle d'alliance ou d'open innovation avec l'industrie au sens large est essentiel, et doit être précoce dans le mécanisme de mise en place des cohortes.

\section{Virginie Fontaine}

Dans le cadre des programmes d'investissements d'avenir, de nombreux appels à projets ont été lancés. L'un d'eux, géré par Bpifrance Financement, porte sur la structuration de filières. Nous n'avons pas encore reçu de projets sur la structuration de la filière des maladies rares. Cet appel à projets s'est aussi ouvert aux platesformes, puisque l'appel à projets «plates-formes mutualisées d'innovation » (PFMI) a été arrêté.

\section{Robert Mamoun, Ciloa, Directeur de recherche Inserm} Comment s'équilibre la mission de service public de la Caisse des dépôts et consignations entre sa fonction de capital-risque et sa fonction de mission publique de développement de biotechnologies et de médicaments dans le cadre des maladies rares?

\section{Laurent Arthaud}

En tant qu'investisseur, il nous est demandé de ne pas investir à perte. Notre portefeuille doit donc être équilibré et nous assurer de dégager un certain bénéfice, aussi modeste soit-il (nous ne nous fixons pas un TRI à $15 \%$ par an). En outre, nous investissons dans les sociétés sur le long terme.

\section{LIENS D'INTÉRÊT}

Les auteurs déclarent n'avoir aucun lien d'intérêt concernant les données publiées dans cet article. 